\title{
The microscopic magnetisation of the superconductor $\mathrm{PuCoGa}_{5}$
}

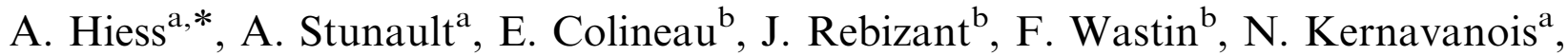 \\ G.J. McIntyre ${ }^{a}$, M.T. Fernandez-Diaz ${ }^{\mathrm{a}}$, E. Lelièvre-Berna ${ }^{\mathrm{a}}$, J.A. Paixão ${ }^{\mathrm{c}}$, G.H. Lander ${ }^{\mathrm{b}}$ \\ ${ }^{a}$ Institut Laue Langevin, BP 156, 38042 Grenoble, France \\ ${ }^{\mathrm{b}}$ European Commission, JRC-Institute for Transuranium Elements, Postfach 2340, 76125 Karlsruhe, Germany \\ ${ }^{\mathrm{c}}$ Physics Department, University of Coimbra, Coimbra 3004-516, Portugal
}

Available online 3 November 2006

\begin{abstract}
The intermetallic compound $\mathrm{PuCoGa}_{5}$ is a superconductor with a surprisingly high critical temperature of $T_{\mathrm{sc}}=18 \mathrm{~K}$ in which the superconductivity has been suggested to arise from magnetic fluctuations. We investigated the microscopic magnetic properties by polarised neutron diffraction on a single crystal of ${ }^{242} \mathrm{PuCoGa}_{5}$. An induced moment is observed on both the Pu and the Co sites. The total magnetisation is significantly smaller than previously reported in agreement with recent magnetisation measurements. This result challenges the currently discussed models for the superconductivity in this weakly magnetic compound.

(C) 2006 Elsevier B.V. All rights reserved.
\end{abstract}

PACS: $71.27+\mathrm{a} ; 74.20 . \mathrm{Mn} ; 75.25 .+\mathrm{z} ; 75.40 . \mathrm{Cx}$

Keywords: Magnetic superconductor; Neutron diffraction; Strongly correlated electron system

\section{Introduction}

$\mathrm{PuCoGa}_{5}$ is one of several isostructural compounds for which superconductivity has been observed. It is a superconductor with a critical temperature of $T_{\mathrm{sc}}=18 \mathrm{~K}$ and an upper critical field $B_{\mathrm{c} 2}$ estimated to exceed $70 \mathrm{~T}$; both parameters are records in this class of intermetallic strongly correlated electron systems [1]. The superconductivity is thought to be unconventional and carried by strongly correlated electrons involving the $5 \mathrm{f}$ electrons of plutonium [2]. No magnetic order has been observed in $\mathrm{PuCoGa}_{5}$ down to $T=1 \mathrm{~K}$, the lowest temperature measured. Those measurements suggest that the bulk magnetic susceptibility follows a modified Curie-Weiss behaviour in the normal state with an effective moment $\mu_{\text {eff }}=0.68 \mu_{\mathrm{B}}$. The induced magnetic moment at $T=20 \mathrm{~K}$ has been proposed to correspond to about $\mu=0.006 \mu_{\mathrm{B}}$ per Tesla [1]. To investigate the microscopic magnetic properties further and possibly to shed light on the relevance of the

\footnotetext{
*Corresponding author. Tel.: + 33476207 165; fax: + 33476483906 .

E-mail address: hiess@ill.fr (A. Hiess).

$U R L:$ http://www.ill.fr.
}

magnetism for the superconductivity, we report here on experiments using polarised neutron diffraction. This experimental technique is sensitive to periodically arranged magnetic moments only and is insensitive to any magnetic contribution from random impurities or the sample mount.

\section{Experimental}

$\mathrm{PuCoGa}_{5}$ crystallises in the $\mathrm{P} 4 / \mathrm{mmm}$ tetragonal crystal structure. The neutron absorption cross section of the most common isotope ${ }^{239} \mathrm{Pu}$ is too high for magnetic neutron scattering experiments. The present experiments were possible thanks to a crystal of sufficient size $(160 \mathrm{mg})$ grown at JRC-ITU, Karlsruhe with the rare ${ }^{242} \mathrm{Pu}$ isotope. It was grown from the melt using ${ }^{242} \mathrm{PuSb}$ instead of metallic ${ }^{239} \mathrm{Pu}$ as starting material. For this reason the crystals contain traces of $\mathrm{Sb}(<1 \%)$ and the superconducting transition temperature is slightly reduced to about $T=15 \mathrm{~K}$. Bulk properties in the normal state such as specific heat and magnetisation do not differ with respect to crystals grown from metallic ${ }^{239} \mathrm{Pu}$ at ITU [3]. 
Crystallographic parameters, as well as the relevant corrections for absorption and extinction, were determined at $T=25 \mathrm{~K}$ by measuring about 600 nuclear reflections with a wavelength of $\lambda=0.84 \AA$ and about 1000 nuclear reflections with a wavelength of $\lambda=0.43 \AA$ using the hot neutron diffractometer D9 at the Institut Laue Langevin (ILL).

The polarised neutron scattering experiments were performed at $\lambda=0.84 \AA$ on the polarised neutron diffractometer D3, also at the ILL. The sample was mounted inside a segmented vertical field cryomagnet with the field of $10 \mathrm{~T}$ applied parallel to the $b$-axis. About 20 flipping ratios $R=I^{+} / I^{-}$were measured at $T=25 \mathrm{~K}$ [4]. From these the magnetic structure factors were extracted using the crystallographic parameters from the D9 experiment. The magnetic structure factors are the Fourier components of the magnetisation density. The magnetisation density map has been reconstructed by Fourier inversion (Fig. 1). Induced magnetic moments are clearly visible on both the $\mathrm{Pu}$ and the Co sites. The uncertainty due to the experimental error and the limited number of measured reflections corresponds to about two contour lines.

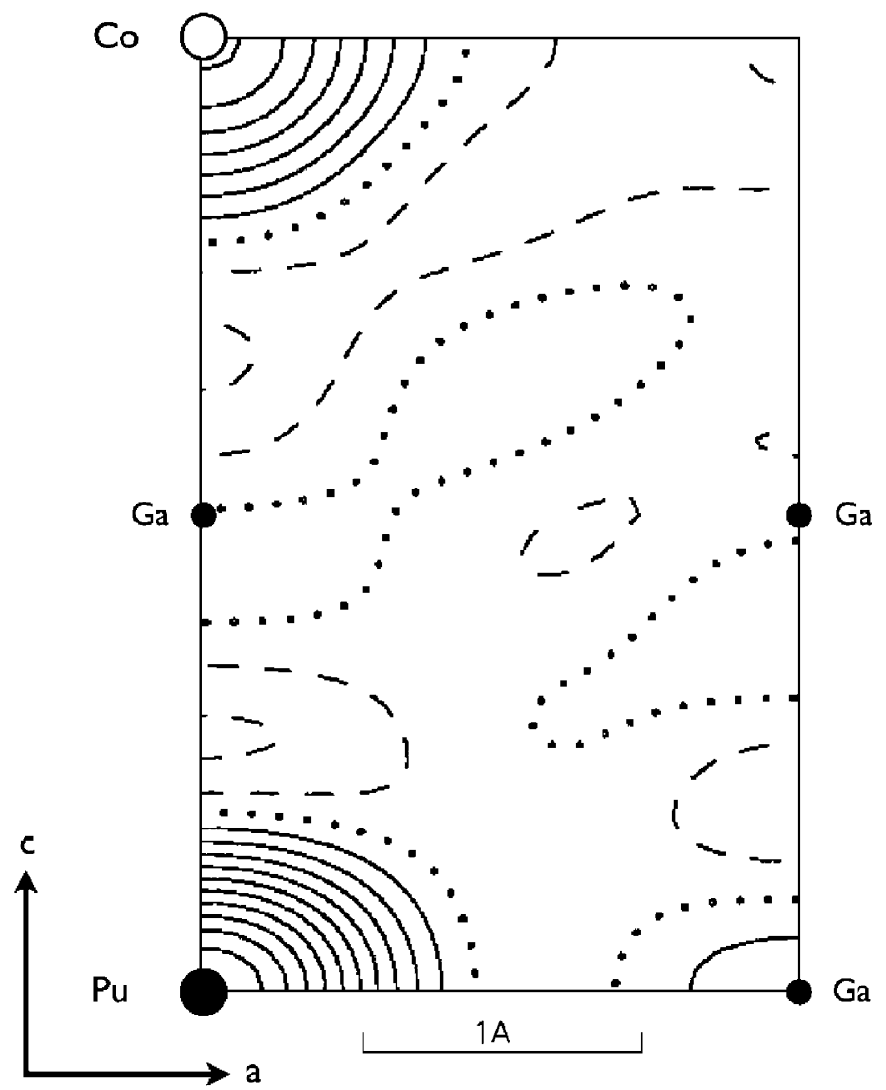

Fig. 1. Magnetisation density of $\mathrm{PuCoGa}_{5}$ projected on the $a c$ plane reconstructed by Fourier inversion from the polarised neutron diffraction data taken at $T=25 \mathrm{~K}$ and an external magnetic field $\mu_{0} H=10 \mathrm{~T}$. Positive, zero and negative magnetisation density is indicated by the solid, dotted and dashed contours, respectively; contour spacing $0.0005 \mu_{\mathrm{B}} / \AA^{2}$.

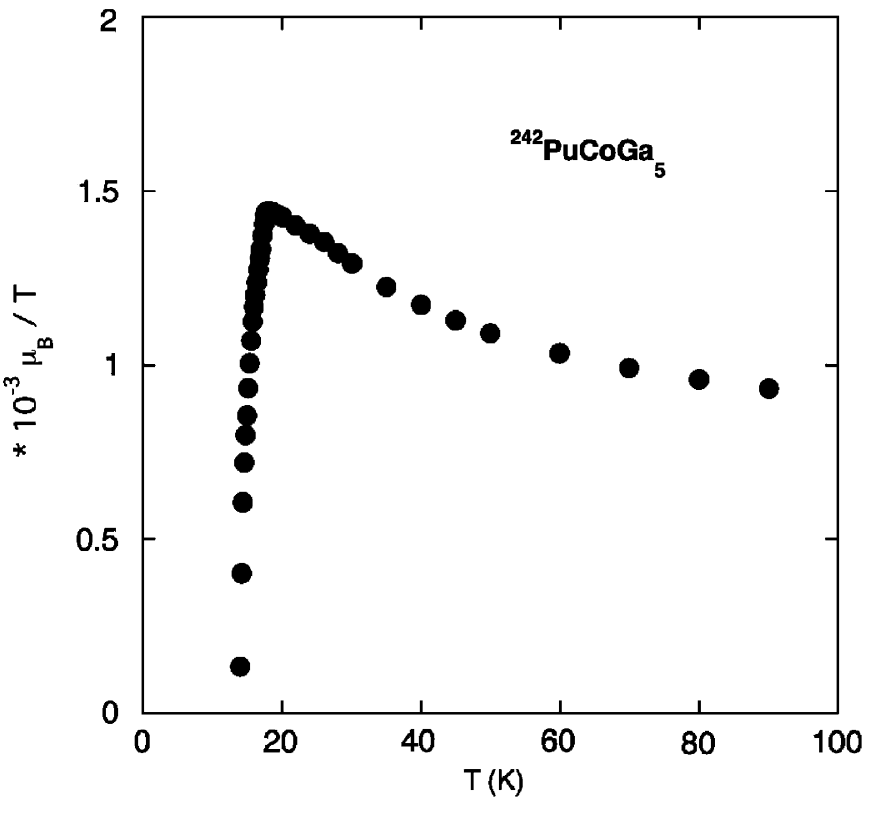

Fig. 2. Induced magnetic moment per Tesla $v$ s. temperature as deduced from bulk magnetisation measurements on single crystals of $\mathrm{PuCoGa}_{5}$ with a magnetic field $\mu_{0} H=7 \mathrm{~T}$. The sharp drop at low temperatures marks the onset of superconductivity.

To get more quantitative information the data were fitted assuming moments on both sites and using the conventional dipole form factors for $\mathrm{Co}$ and $\mathrm{Pu}$. Those fits indicate an induced moment per Tesla of $0.0004( \pm 0.0001)$ and $0.0002( \pm 0.0001) \mu_{\mathrm{B}}$ on the $\mathrm{Pu}$ and Co sites, respectively. Even considering the error bars, the total induced moment does not exceed $0.001 \mu_{\mathrm{B}}$ per Tesla.

\section{Discussion}

The polarised neutron diffraction experiments indicate an induced moment significantly lower than previously reported [1], but they are consistent with recent bulk magnetisation measurements (Fig. 2) on similar samples [3]. In the latter measurements an induced moment of about $0.0014 \mu_{\mathrm{B}}$ per Tesla was observed at $T=25 \mathrm{~K}$. The agreement is quite reasonable considering that the bulk measurements rely strongly on corrections for the sample mount, and are sensitive to impurities, while the neutron experiments are sensitive to the periodic arrangement of the magnetic moment in the crystallographic lattice only.

We therefore conclude that the intrinsic magnetisation of $\mathrm{PuCoGa}_{5}$ is lower than previously reported.

This result is difficult to reconcile with ferromagnetic $(q=0)$ magnetic fluctuations. As recent NMR experiments [2] underline the presence of magnetic fluctuations above $T_{\text {sc }}$ inelastic neutron scattering experiments are now underway to search for possible magnetic fluctuations at $q \neq 0$ in this fascinating compound. 


\section{Acknowledgements}

We thank the health physics and the technical services of the ILL for their help in setting up these experiments using transuranium samples. We acknowledge the help of $\mathbf{J}$. Brown, ILL, and E. Ressouche, CEA/Grenoble, on the Fourier inversion of the polarised neutron data.

\section{References}

[1] J.L. Sarrao, et al., Nature 420 (2002) 297.

[2] N.J. Curro, et al., Nature 434 (2005) 622 and references therein.

[3] E. Colineau, et al., in preparation.

[4] For details on polarised neutron diffraction see: W.G. Williams, Polarised Neutrons, Oxford University Press, Oxford, 1988. 